Short Paper

\title{
Isolation of Microsatellite Loci from Japanese Flounder Paralichthys olivaceus and Detection of PCR Fragments with Simple Non-RI Methods
}

\author{
Motohiro Takagi, ${ }^{* 1, \uparrow 1}$ Kazunori Yoshida, ${ }^{* 1}$ and Nobuhiko Taniguchi ${ }^{* 1, \nmid 2, \uparrow 3}$ \\ ${ }^{*}$ 'Department of Cultural Fisheries, Faculty of Agriculture, Kochi University, \\ Monobe, Nankoku, Kochi 783-8502, Japan
}

(Received May 29, 1998)

Key words: microsatellite, Japanese flounder, Paralichthys orivaceus, genetic variability, NonRI, ethidium bromide, silver staining

In recent years, Japanese flounder Paralichthys olivaceus has been artificially propagated and released into many of the coastal waters of Japan for stock enhancement. It is thus necessary to investigate the genetic diversity of the wild population and the hatchery stock in relation to the genetic conservation of the wild population. Microsatellites have been shown to be highly polymorphic in nature, and their accuracy for assaying has made them the best of choice for high resolution population analysis. ${ }^{1)}$ However, the difficulty of the method of detection of PCR amplified fragments with radioisotope (RI), automatedDNA sequencer and chemiluminescent has prevented the microsatellite from being widely used for fish population and breeding studies.

In the present study, we report the isolation and characterization of five microsatellite loci from the Japanese flounder and examine the detection of PCR fragments with formal Non-RI methods (Ethidium bromide and Silver staining) for convenient routine study.

Samples of the Japanese flounder were collected from Nagasaki Prefecture, and DNA samples were extracted from whole blood and fin clip by standard SDS-phenol/ chloroform procedures ${ }^{2)}$ and stored at $4^{\circ} \mathrm{C}$ prior to PCR analysis.

Primers design to hybridize with unique regions flanking the core sequences of the microsatellites, PCR, PAG electrophoresis, and detection bands with RI were carried out using the methods described by Takagi et al.$^{3)}$ Alleles were sized relative to a M13 sequence rudder. ${ }^{4)}$ Then, PCR amplified fragments of simple Non-RI methods were electrophoresed with $20 \mathrm{bp}$ molecular markers (Takara Inc.), using long gel (e.g. sequence electrophoresis chamber). The electrophoresis carried on non-denaturized polyacrylamide gel $(1 \times$ TBE, $7 \%$ glycerol, $8 \%$ polyacrylamide) at $10 \mathrm{~V} / \mathrm{cm}$ for $16 \mathrm{hrs}$. in I $\times$ TBE buffer $(0.1 \mathrm{M}$ Tris, $0.1 \mathrm{M}$ boric acid, $0.002 \mathrm{M}$ EDTA, pH 8.3). DNA fragments in the gels were stained by standard protocol of ethidium bromide ${ }^{5)}$ (Et.Br.) and photographed on a UV transilluminator. Silver staining was performed according to manufacture's protocol (Wako Inc.).

Primer sequences, annealing temperature, number of alleles, amplified product size range, and heterozygosities for the isolated 5 loci are summarized in Table 1 . The 5 microsatellite loci were all highly polymorphic, while the degree of variability was different, at each locus. Pol-2* had the lowest number of alleles (6) and heterozygosity

Table 1. Primer sequence, allele size, heterozygosity of Japanese flounder microsatellite loci detected by PCR amplification

\begin{tabular}{|c|c|c|c|c|c|c|c|}
\hline Locus & motif*a & Primer sequence $\left(5^{r}-3^{\prime}\right)^{* \mathrm{~b}}$ & $\begin{array}{c}\text { Annealing } \\
\text { temp. }\end{array}$ & $\begin{array}{l}\text { No. of } \\
\text { samples }\end{array}$ & $\begin{array}{l}\text { No. of } \\
\text { alleles }\end{array}$ & Size $(b p)^{* d}$ & $\begin{array}{l}\text { Heterozygosity } \\
(\mathrm{He})^{*_{c}}\end{array}$ \\
\hline \multirow[t]{2}{*}{ Pol-1 } & (GT) & F: TAGGAGGATTGATAGGAT & 53 & 38 & 36 & $143-245$ & 0.952 \\
\hline & & R: CCCGACTTTTAGATGTTC & & & & & \\
\hline \multirow[t]{2}{*}{ Pol-2* } & (GT) & F: AGGATTTAGCCAAGGTCA & 53 & 40 & 6 & $110-124$ & 0.689 \\
\hline & & R: ACATCAAGCCAAGAGAAG & & & & & \\
\hline \multirow[t]{2}{*}{ Pol-3* } & (GT) & F: TTTGTGTTGTAGTTAGTCTG & 50 & 38 & 22 & $116-178$ & 0.910 \\
\hline & & R: GTTGATTGTAGAGTGGTGTG & & & & & \\
\hline \multirow[t]{2}{*}{ Pol-4* } & $(\mathrm{GT})$ & F: GTGTGCTCTGGGCTTTTG & 50 & 39 & 28 & $91-197$ & 0.935 \\
\hline & & R: TTACCTGTCCTGTTACTC & & & & & \\
\hline \multirow[t]{2}{*}{ Pol-5* } & (CA) & F: AACTGCTCCTTGAACTGC & 53 & 39 & 21 & $109-175$ & 0.888 \\
\hline & & R: GGAAGCATCGACTCTCAT & & & & & \\
\hline
\end{tabular}

$*_{\mathrm{a}}=$ Core repeat motif from cloned Paralichthys otivaceus sequence. ${ }^{*} \mathrm{~b}=\mathrm{F}$ and $\mathrm{R}$ refer to forward and reverse primer sequence respectively. ${ }^{*} \mathrm{c}=\mathrm{PCR}$ annealing temperatures were optimized for Paralichtys olivaceus. ${ }^{*}{ }^{*}=$ Estimated size of the PCR fragment when compared with M13 sequence fragmentes of known length. ${ }^{*}=$ Estimated heterozygosity.

t1 Present address: Ono Limnological Station, National Fisheries University, Ono, Ube, Yamaguchi 754-1311, Japan.

t2 Present address: Labolatory of Applied Population Genetics, Graduate School of Agriculture, Tohoku University, Amamiya-cho, Aoba, Sendai 981-8555, Japan.

${ }^{+3}$ To whom correspondence should be addressed. 
Table 2. Allele frequencies of five microsatellite loci of Japanese flounder, Paralichthys olivaceus from Nagasaki Prefecture

\begin{tabular}{|c|c|c|c|c|c|c|c|c|c|}
\hline Allele & Pol-1* & Allele & Pol-2* & Allele & Pol-3* & Allele & Pol-4* & Allele & Pol-5* \\
\hline 143 & 0.026 & 110 & 0.350 & 116 & 0.013 & 92 & 0.013 & 109 & 0.013 \\
\hline 145 & 0.026 & 112 & 0.400 & 122 & 0.026 & 118 & 0.026 & 121 & 0.244 \\
\hline 147 & 0.013 & 114 & 0.075 & 128 & 0.026 & 122 & 0.026 & 123 & 0.128 \\
\hline 149 & 0.026 & 116 & 0.150 & 130 & 0.171 & 124 & 0.013 & 125 & 0.051 \\
\hline 151 & 0.105 & 122 & 0.125 & 132 & 0.132 & 126 & 0.026 & 127 & 0.103 \\
\hline 153 & 0.013 & 124 & 0.125 & 134 & 0.079 & 128 & 0.026 & 129 & 0.077 \\
\hline 155 & 0.040 & & & 140 & 0.066 & 132 & 0.026 & 131 & 0.013 \\
\hline 157 & 0.026 & & & 142 & 0.118 & 134 & 0.180 & 133 & 0.103 \\
\hline 159 & 0.066 & & & 146 & 0.079 & 138 & 0.013 & 135 & 0.026 \\
\hline 161 & 0.026 & & & 148 & 0.040 & 140 & 0.026 & 139 & 0.026 \\
\hline 163 & 0.040 & & & 150 & 0.013 & 142 & 0.051 & 141 & 0.026 \\
\hline 165 & 0.013 & & & 152 & 0.026 & 144 & 0.013 & 145 & 0.013 \\
\hline 167 & 0.066 & & & 154 & 0.040 & 148 & 0.064 & 147 & 0.026 \\
\hline 169 & 0.026 & & & 156 & 0.013 & 152 & 0.051 & 151 & 0.013 \\
\hline 171 & 0.053 & & & 158 & 0.026 & 154 & 0.064 & 153 & 0.013 \\
\hline 173 & 0.053 & & & 160 & 0.056 & 156 & 0.051 & 155 & 0.013 \\
\hline 175 & 0.013 & & & 162 & 0.013 & 158 & 0.064 & 157 & 0.026 \\
\hline 177 & 0.013 & & & 164 & 0.013 & 160 & 0.039 & 163 & 0.013 \\
\hline 179 & 0.053 & & & 170 & 0.013 & 162 & 0.026 & 167 & 0.026 \\
\hline 181 & 0.026 & & & 172 & 0.013 & 164 & 0.026 & 171 & 0.039 \\
\hline 183 & 0.013 & & & 178 & 0.013 & 166 & 0.026 & 175 & 0.013 \\
\hline 185 & 0.238 & & & 184 & 0.013 & 168 & 0.147 & & \\
\hline S & & & & & & $S$ & & & \\
\hline 245 & & & & & & 198 & & & \\
\hline
\end{tabular}

(0.689) of the five loci. Pol-1* ${ }^{*} \mathrm{Pol}-3^{*}, \mathrm{Pol}-4^{*}$ and Pol-5* were highly polymorphic in both allele number and heterozygosity, as observed in the total number of alleles per locus (21-36), and a high degree of heterozygosity ranging from 0.888 to 0.952 . Allele frequencies of the five microsatellites for samples from Nagasaki Prefecture are shown in Table 2. At locus $\mathrm{Pol}-2^{*}$, the frequencies of major alleles 110 and 112 were 0.350 and 0.400 . At loci Pol $-\mathrm{I}^{*}$ and $\mathrm{Pol}-4^{*}$, allele frequencies were overall low with the most common allele only having frequency under 0.100. At loci Pol-3* and Pol-5*, were somewhat imtermediate with the major allele having a frequency at 0.171 and 0.244 . These microsatellites will be used widely in further population analysis and monitoring genetic change of brood stocks in fisheries and aquaculture due to their highly polymorphic nature.

Figure 1 shows the Microsatellite electropherogram obtained using the primer Pol 2 locus with RI labeled, Et. Br. and Silver staining methods. Allele identification was easy for each of the alleles detected every $2 \mathrm{bp}$ between $20 \mathrm{bp}$ molecular markers according to Et.Br. and Silver staining methods. Et.Br. and Silver staining methods were compared with band patterns and allele size of RI method. When using the Et.Br. method, density of homozygous bands were more distinct than that of the heterozygous bands. Furthermore, the silver staining method showed higher resolution than the Et.Br. method. As a result of the high resolution of the silver staining method, shadow bands were detected. Moreover, real bands were very distinct, so misreading could be avoided in allele identification. These results suggest that the Et.Br. and the silver staining methods are not only useful in detection of microsatellite PCR fragment under Non-RI conditions for high resolution but also economical reasons.

The authors are grateful to Prof. M. Tanaka, Kyoto University, for providing fish samples. The present study was supported in part by a
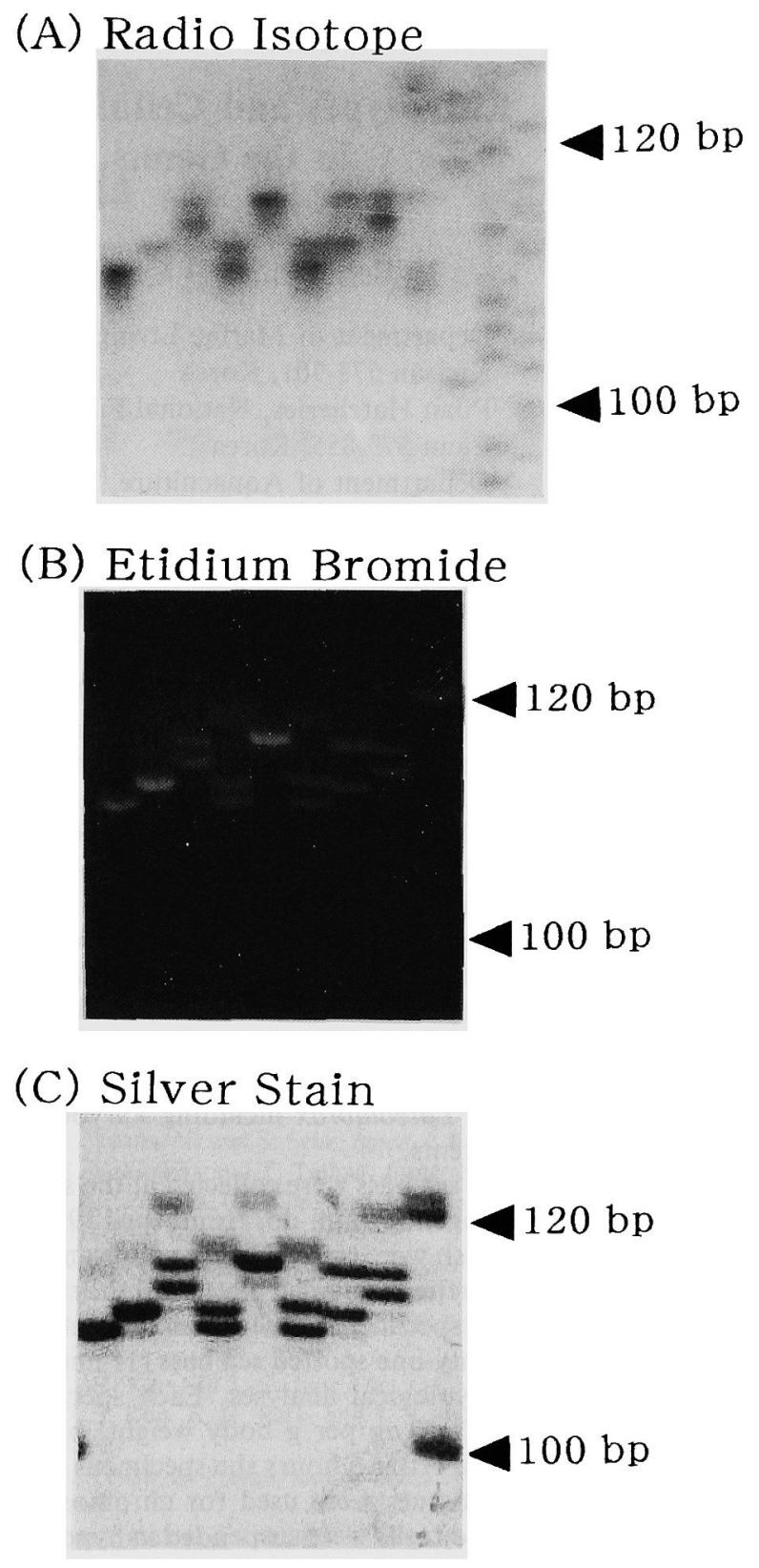

Fig. 1. Banding pattern of electropherogram of $\mathrm{Pol}-2^{*}$ microsatellite locus.

(A) Radioisotope detection. The size standard is a sequencing rudder of M13 mp18. (B) Ethidium bromide staining. (C) Silver staining. The size standard of (B) and (C) are $20 \mathrm{bp}$ molecular markers.

grant from the Fisheries Agency, Japan to N. Taniguchi.

\section{References}

1) A. Estoup, P. Presa, F. Krieg, D. Vaiman, and R. Guyomard: Herediry, 71, 488-496 (1993).

2) A. S. Harris, S. Bieger, R. W. Doyle, and J. M. Wright: Aquaculture, 92, 157-163 (1991).

3) M. Takagi, N. Taniguchi, D. Cook, and R. W. Doyle: Fisheries Sci., 63, 199-204 (1997).

4) C. Yanisch-Perron, J. Vieira, and J. Messing: Gene, 33, 103-119 (1985).

5) J. Sambrook, E. F. Fritsch, and T. Maniatis: Molecular Cloning, Cold Springer Harbor Loboratory Press, New York, 1989, pp. 6.44. 